\title{
Quenching phenomenon for a non-Newtonian filtration equation with singular boundary flux
}

Ying Yang*

*Correspondence:

yiyiying729@163.com School of Mathematics and

Statistics, Shenzhen University,

Shenzhen, 518060, P.R. China

\begin{abstract}
This paper is concerned with the quenching phenomenon for the one-dimensional non-Newtonian filtration equation with both source term and Neumann boundary condition. With two different kinds of initial data, we prove that the solution must quench in a finite time and the time derivative blows up at a quenching point. The corresponding quenching rate and a lower bound for the quenching time are also obtained.
\end{abstract}

MSC: 34B15; 35K55; 35K65

Keywords: non-Newtonian filtration equation; singular boundary flux; finite time quenching; quenching rate

\section{Introduction}

In this paper, we study the following problem:

$$
\begin{aligned}
& u_{t}=\left(\left|u_{x}\right|^{p-2} u_{x}\right)_{x}+(1-u)^{-h}, \quad 0<x<1, t>0, \\
& u_{x}(0, t)=0, \quad u_{x}(1, t)=-u^{-q}(1, t), \quad t>0, \\
& u(x, 0)=u_{0}(x), \quad 0 \leq x \leq 1,
\end{aligned}
$$

where $p \geq 2, h, q$ are positive constants. $u_{0}(x):[0,1] \rightarrow(0,1)$ and satisfies some compatibility conditions. Equation (1.1) is known as the classical non-Newtonian filtration equation that incorporates the effects of nonlinear reaction source and nonlinear boundary outflux. The quenching behavior describes the phenomenon that there exists a finite time $T$ such that the solution $u(x, t)$ of the problem (1.1) satisfy

$$
\lim _{t \rightarrow T^{-}} \max \{u(x, t): 0 \leq x \leq 1\} \rightarrow 1 \text { or } \quad \lim _{t \rightarrow T^{-}} \min \{u(x, t): 0 \leq x \leq 1\} \rightarrow 0
$$

In 1975, Kawarada [1] first studied the quenching phenomenon for the semilinear heat equation $u_{t}=u_{x x}+(1-u)^{-1}$. He obtained the results that, when the solution reaches level $u=1$, the reaction term and the time derivative blow up. Since then, quenching phenomena for semilinear parabolic equations have been studied by many researchers; see for ex-

(c) 2015 Yang. This article is distributed under the terms of the Creative Commons Attribution 4.0 International License (http://creativecommons.org/licenses/by/4.0/), which permits unrestricted use, distribution, and reproduction in any medium, provided you give appropriate credit to the original author(s) and the source, provide a link to the Creative Commons license, and indicate if changes were made. 
amples [1-6] and the references therein. Quenching phenomenon is dependent on the singular term of the model. Different singular terms cause the problem may occur quenching phenomenon at different levels. Recently, more and more researchers have focused on the quenching phenomenon for parabolic problem with two nonlinear heat sources [7-12]. For example, Chan and Yuen [7] investigated the problem with two nonlinear boundary outfluxes:

$$
\begin{aligned}
& u_{t}=u_{x x}, \quad 0<x<a, 0<t<T, \\
& u_{x}(0, t)=(1-u(0, t))^{-p}, \quad u_{x}(a, t)=(1-u(a, t))^{-q}, \quad 0<t<T, \\
& u(x, 0)=u_{0}(x), \quad 0 \leq x \leq a,
\end{aligned}
$$

where $a, p, q>0$. The authors proved that the solution quenches only at $x=a$, meanwhile, the time derivative $u_{t}$ blows up. Moreover, making use of the positive steady states, they have given criteria for nonquenching and quenching. It is worth mentioning the work by Selcuk and Ozalp [8], who considered the problem

$$
\begin{aligned}
& u_{t}=u_{x x}+(1-u)^{-p}, \quad 0<x<1,0<t<T, \\
& u_{x}(0, t)=0, \quad u_{x}(a, t)=-u(1, t)^{-q}, \quad 0<t<T, \\
& u(x, 0)=u_{0}(x), \quad 0 \leq x \leq 1,
\end{aligned}
$$

where $p, q>0$. They showed that quenching occurs only at $x=0$ in finite time and they estimated the bounds of the quenching rate and a lower bound for the quenching time.

On the other hand, as is well known, the singular or degenerate parabolic equations have the property of finite speed of propagation, which are more consistent with biological phenomena in the real world. So, it should be more reasonable to discuss some nonlinear diffusion models. There is a natural question if quenching phenomenon may occur for singular or degenerate equations. Few works were concerned with singular or degenerate parabolic equations [13-19], where only models with one nonlinear source are studied. In [13-15], the authors studied a nonlinear equation with homogeneous boundary conditions. In [16-19], a nonlinear model with nonlinear boundary conditions was discussed. It was Nie et al. [14] who considered quenching for a singular and degenerate quasilinear diffusion equation as follows:

$$
\begin{aligned}
& x^{q} \frac{\partial u}{\partial t}-\frac{\partial^{2} u^{m}}{\partial x^{2}}=f\left(u^{m}\right), \quad(x, t) \in(0, a) \times(0, T), \\
& u(0, t)=0=u(a, t), \quad t \in(0, T), \\
& u(x, 0)=0, \quad x \in(0, a) .
\end{aligned}
$$

Here $a>0, q \in \mathbb{R}, m \geq 1$. They established the existence of a critical length $a_{*}$ and proved that the solution exists globally if $0<a<a_{*}$, while the solution quenches if $a>a_{*}$. They also investigated the set of the quenching points and the blowing up of $u_{t}$. Deng and $\mathrm{Xu}$ [16] studied nonlinear diffusion equation with a singular boundary condition and investigated finite time quenching for the solution. They also gave the quenching set and quenching rate near the quenching time. 
However, as far as we know, there are very few papers concerned with the quenching phenomenon for singular or degenerate parabolic problem with two nonlinear heat sources, even if the linear diffusion equation holds. Obviously, in the model (1.1), the internal source $(1-u)^{-h}$ and the boundary flux $u^{-q}$ exist, both of which may become singular in some finite time, if the solution reach level $u=1$ or $u=0$, respectively. In the present paper, we will discuss these two cases by imposing conditions $\left(\mathrm{A}_{1}\right)-\left(\mathrm{A}_{4}\right)$ upon the initial datum, which are give below. First of all, motivated by the work of [8], in Section 2, we will study the quenching phenomenon for the solution reaching the level $u=1$. We will prove that quenching occurs in finite time under condition $\left(\mathrm{A}_{1}\right)$ and the only quenching point is $x=0$ under conditions $\left(\mathrm{A}_{1}\right)$ and $\left(\mathrm{A}_{2}\right)$. Furthermore, $u_{t}$ blows up at quenching time is discussed. Then the bounds for the quenching rate and the lower bound for the quenching time are estimated. Second, in Section 3, we will do research on the quenching phenomenon for the solution reaching the level $u=0$ under conditions $\left(\mathrm{A}_{2}\right)$ and $\left(\mathrm{A}_{4}\right)$. It will be shown that the solution quenches in finite time and $u_{t}$ blows up at quenching time at the only quenching point $x=1$. Finally, we will give bounds on the quenching rate.

Furthermore, in this paper, we need the following hypotheses:

$\left(\mathrm{A}_{1}\right) \quad\left(\left|u_{0}^{\prime}(x)\right|^{p-2} u_{0}^{\prime}(x)\right)^{\prime}+\left(1-u_{0}(x)\right)^{-h} \geq 0 ;$

$\left(\mathrm{A}_{2}\right) \quad u_{0}^{\prime}(x) \leq 0$

$\left(\mathrm{A}_{3}\right) u_{0}^{\prime}(x) \leq-x u_{0}^{-q}(x)$

(A $\left.\mathrm{A}_{4}\right)\left(\left|u_{0}^{\prime}(x)\right|^{p-2} u_{0}^{\prime}(x)\right)^{\prime}+\left(1-u_{0}(x)\right)^{-h} \leq 0$.

\section{Quenching phenomenon for the solution reaching the level $u=1$}

In this section, we study the quenching phenomenon for the problem (1.1) under the conditions $\left(\mathrm{A}_{1}\right)$ and $\left(\mathrm{A}_{2}\right)$. Due to the degeneracy of the equation, the classical solutions might not exist and the weak solution should be considered. However, for simplicity of our arguments, we assume that the solution is appropriately smooth, since we may consider some approximate boundary and initial value conditions.

\subsection{Quenching on the boundary and blow-up of $u_{t}$}

In this section, we prove the solution quenches in finite time and blowing up of $u_{t}$ at the only quenching point $x=0$.

Remark 2.1 The assumptions $\left(\mathrm{A}_{1}\right)$ and $\left(\mathrm{A}_{2}\right)$ on $u_{0}(x)$ are proper. For example, for $p=2$, $h=9$, and $q=\log _{30 / 7} 3$, we can choose $u_{0}(x)=0.9-\frac{3}{2} x^{4.5}$, which satisfies $\left(\mathrm{A}_{1}\right),\left(\mathrm{A}_{2}\right)$, and compatibility conditions.

In the following, we discuss the properties of the solution to the problem (1.1).

Lemma 2.1 Assume that $\left(\mathrm{A}_{1}\right),\left(\mathrm{A}_{2}\right)$ hold and the solution $u$ of the problem (1.1) exists in $\left(0, T_{0}\right)$ for some $T_{0}>0$. Then $u \in C^{2,1}\left((0,1] \times\left(0, T_{0}\right)\right)$ with $u_{x}(x, t)<0$ and $u_{t}(x, t) \geq 0$ in $(0,1] \times\left(0, T_{0}\right)$.

Proof Let $v=u_{x}$. Then $v$ satisfies

$$
\begin{aligned}
& v_{t}=\left(|v|^{p-2} v\right)_{x x}+h(1-u)^{-h-1} v, \quad 0<x<1,0<t<T_{0}, \\
& v(0, t)=0, \quad v(1, t)=-u^{-q}(1, t), \quad 0<t<T_{0}, \\
& v(x, 0)=u_{0}^{\prime}(x), \quad 0 \leq x \leq 1 .
\end{aligned}
$$


Using the maximum principle, we have $v<0$, that is, $u_{x}(x, t)<0$ in $(0,1] \times\left(0, T_{0}\right)$. Then it is easy to see that the problem $(2.1)$ is nondegenerate in $(0,1] \times\left(0, T_{0}\right)$. So $u_{x}$ is a classical solution of (2.1).

On the other hand, setting $w=u_{t}$, then $w$ solves the following:

$$
\begin{aligned}
& w_{t}=(p-1)\left(\left|u_{x}\right|^{p-2} w_{x}\right)_{x}+h(1-u)^{-h-1} w, \quad 0<x<1,0<t<T_{0}, \\
& w_{x}(0, t)=0, \quad w_{x}(1, t)=q u^{-q-1}(1, t) w(1, t), \quad 0<t<T_{0}, \\
& w(x, 0)=(p-1)\left|u_{0}^{\prime}(x)\right|^{p-2} u_{0}^{\prime \prime}(x)+\left(1-u_{0}(x)\right)^{-h}, \quad 0 \leq x \leq 1 .
\end{aligned}
$$

Utilizing the maximum principle, one shows that $u_{t}(x, t) \geq 0$ in $(0,1] \times\left(0, T_{0}\right)$. Therefore, the solutions of the problem (1.1) $u \in C^{2,1}\left((0,1] \times\left(0, T_{0}\right)\right)$ and they satisfy $u_{x}(x, t)<0$ and $u_{t}(x, t) \geq 0$ in $(0,1] \times\left(0, T_{0}\right)$.

Now, we are in a position to show the quenching result.

Theorem 2.1 Assume that $\left(\mathrm{A}_{1}\right)$ and $\left(\mathrm{A}_{2}\right)$ hold. Then there exists a finite time $T$, such that every solution of (1.1) quenches in this time, and the only quenching point is $x=0$.

Proof The maximum principle leads to $0<u(\cdot, t)<1$ for all $t$ in the existence interval. Taking advantage of the assumption $\left(\mathrm{A}_{1}\right)$, we have

$$
\alpha=-u^{-q(p-1)}(1,0)+\int_{0}^{1}(1-u(x, 0))^{-h} d x>0 .
$$

Denote $A(t)=\int_{0}^{1}(1-u(x, t)) d x$. By Lemma 2.1 , it is easy to see that

$$
A^{\prime}(t)=-\int_{0}^{1} u_{t}(x, t) d x=u^{-q(p-1)}(1, t)-\int_{0}^{1}(1-u(x, t))^{-h} d x \leq-\alpha .
$$

Thus $A(t) \leq A(0)-\omega t$, which means that $A\left(t_{0}\right)=0$ for some $t_{0}>0$. In addition, since $u_{x}<0$ for $0<x \leq 1$, we can see that there exists $T\left(0<T<t_{0}\right)$ such that $\lim _{t \rightarrow T^{-}} u(0, t)=$ 1. By means of the singular nonlinearity in the source, $u$ must occur quenching on the boundary $x=0$. Here and below, we use $T$ to denote the quenching time of the solutions $u$. In the following, we only need to show that the solutions $u$ cannot take place quenching in $(0,1] \times(\eta, T)$ for some $\eta(0<\eta<T)$.

Denote

$$
B(x, t)=u_{x}+\varepsilon\left(b_{2}-x\right) \quad \text { in }\left(b_{1}, b_{2}\right) \times[\eta, T),
$$

where $b_{2} \in(0,1], b_{1} \in\left(0, b_{2}\right)$, and $\varepsilon$ is a positive constant to be specified later. Since $u_{x}(x, t)<0$ in $(0,1] \times[0, T), B(x, t)$ satisfies

$$
\begin{aligned}
B_{t} & -(p-1)\left|u_{x}\right|^{p-2} B_{x x} \\
& =-(p-1)(p-2)\left|u_{x}\right|^{p-3}\left(B_{x}+\varepsilon\right)^{2}+h(1-u)^{-h-1} u_{x}<0, \quad \text { for }(x, t) \in\left(b_{1}, b_{2}\right) \times[\eta, T) .
\end{aligned}
$$


Hence, according to the maximum principle, $B(x, t)$ cannot attain a positive interior maximum. Further, on the parabolic boundary, since $u_{x}(x, t)<0$ in $(0,1] \times[0, T)$, choosing $\varepsilon$ small enough, we have

$$
\begin{aligned}
& B\left(b_{1}, t\right)=u_{x}\left(b_{1}, t\right)+\varepsilon\left(b_{2}-b_{1}\right)<0, \quad t \in(\eta, T), \\
& B\left(b_{2}, t\right)=u_{x}\left(b_{2}, t\right)<0, \quad t \in(\eta, T), \\
& B(x, \eta)=u_{x}(x, \eta)+\varepsilon\left(b_{2}-x\right), \quad x \in\left(b_{1}, b_{2}\right) .
\end{aligned}
$$

Making use of the maximum principle, we obtain $B(x, t)<0$, that is,

$$
u_{x}<-\varepsilon\left(b_{2}-x\right), \quad(x, t) \in\left[b_{1}, b_{2}\right] \times[\eta, T) .
$$

Integrating the above inequality with respect to $x$ from $b_{1}$ to $b_{2}$ gives

$$
u\left(b_{2}, t\right)<u\left(b_{1}, t\right)-\frac{\varepsilon\left(b_{2}-b_{1}\right)^{2}}{2}<1-\frac{\varepsilon\left(b_{2}-b_{1}\right)^{2}}{2}<1,
$$

which implies that $u(x, t)<1$ if $0<x \leq 1$.

Theorem 2.2 Assume that $h \geq 1$. Then $u_{t}$ blows up at the quenching point $x=0$.

Proof We prove the theorem by contradiction. Assume that $u_{t}$ is bounded on $[0,1] \times$ $[0, T)$. Then there exists a positive constant $M$ such that $u_{t}<M$. Thus, we have

$$
\left(\left|u_{x}\right|^{p-2} u_{x}\right)_{x}+(1-u)^{-h}<M .
$$

Multiplying the above inequality by $u_{x}$, and integrating with respect to $x$ from 0 to $x$ yield

$$
\ln [1-u(0, t)]>-\frac{p-1}{p}\left|u_{x}\right|^{p}+\ln [1-u(x, t)]+M[u(x, t)-u(0, t)]
$$

for $h=1$ and

$$
\frac{(1-u(0, t))^{-h+1}}{-h+1}>-\frac{p-1}{p}\left|u_{x}\right|^{p}+\frac{(1-u(x, t))^{-h+1}}{-h+1}+M[u(x, t)-u(0, t)]
$$

for $h \neq 1$. It can be seen that the left-hand side tends to negative infinity as $t \rightarrow T^{-}$, while the right-hand side is finite. This completes the proof of Theorem 2.2.

\subsection{Quenching rate and lower bound for the quenching time}

In this section, a bound on the quenching rate is given and a lower bound for the quenching time is obtained. We present the quenching rate in the following:

Theorem 2.3 Assume that $\left(\mathrm{A}_{1}\right),\left(\mathrm{A}_{2}\right)$, and $\left(\mathrm{A}_{3}\right)$ hold. Then there exists a positive constant $C_{1}$ such that

$$
u(0, t) \geq 1-C_{1}(T-t)^{1 /(h+1)}
$$


Proof We define a function $G(x, t)=\left|u_{x}(x, t)\right|^{p-2} u_{x}(x, t)+x^{p-1} u^{-q(p-1)}(x, t)$ in $[0,1] \times[0, T)$. Then $G(x, t)$ solves

$$
\begin{aligned}
G_{t}- & (p-1)\left|u_{x}\right|^{p-2} G_{x x} \\
= & -(p-1)\left|u_{x}\right|^{p-1} h(1-u)^{-h-1}-q(p-1) u^{-q(p-1)-1} x^{p-1}(1-u)^{-h} \\
& -(p-1)^{2}(p-2)\left|u_{x}\right|^{p-2} x^{p-3} u^{-q(p-1)}-2 q(p-1)^{3}\left|u_{x}\right|^{p-1} x^{p-2} u^{-q(p-1)-1} \\
& -(p-1)^{2} q[q(p-1)+1] x^{p-1} u^{-q(p-1)-2}\left|u_{x}\right|^{p},
\end{aligned}
$$

since $u_{x}<0, G(x, t)$ cannot attain a positive interior maximum. On the other hand, it follows from $\left(\mathrm{A}_{3}\right)$ that

$$
G(x, 0)=-\left(u_{x}(x, 0)\right)^{p-1}+x^{p-1} u^{-q(p-1)}(x, 0) \leq 0 .
$$

Also

$$
G(0, t)=0, \quad G(1, t)=0
$$

for $t \in(0, T)$. The maximum principle yields $G(x, t) \leq 0$ for $(x, t) \in[0,1] \times[0, T)$. Therefore

$$
G_{x}(0, t)=\lim _{\sigma \rightarrow 0^{+}} \frac{G(\sigma, t)-\Phi(0, t)}{\sigma}=\lim _{\sigma \rightarrow 0^{+}} \frac{G(\sigma, t)}{\sigma} \leq 0 .
$$

Hence, for $p=2$,

$$
G_{x}(0, t)=u_{x x}(0, t)+u^{-q}(0, t)=u_{t}(0, t)-(1-u(0, t))^{-h}+u^{-q}(0, t) \leq 0
$$

and for $p>2$

$$
G_{x}(0, t)=\left(\left|u_{x}\right|^{p-2} u_{x}\right)_{x}(0, t)=u_{t}(0, t)-(1-u(0, t))^{-h} \leq 0 .
$$

Thus, we get

$$
u_{t}(0, t) \leq(1-u(0, t))^{-h}
$$

Integrating for $t$ from $t$ to $T$, we have

$$
u(0, t) \geq 1-C_{1}(T-t)^{\frac{1}{h+1}}
$$

where $C_{1}=(h+1)^{1 /(h+1)}$. This completes the proof of Theorem 2.3 .

Remark 2.2 According to Theorem 2.3, a lower bound of quenching time $T$ is (1$\left.u_{0}(0)\right)^{h+1} /(h+1)$. As in Remark 2.1, if $u_{0}(x)=0.9-\frac{2}{3} x^{4.5}$, then it can be found that $T=10^{-11}$ for $h=9$.

\section{Quenching phenomenon for the solution reaching the level $u=0$}

In this section, we investigate the quenching phenomenon for the problem (1.1) under the conditions $\left(\mathrm{A}_{2}\right)$ and $\left(\mathrm{A}_{4}\right)$. 


\subsection{Quenching on the boundary and blow-up of $u_{t}$}

In this section, we prove the solution quenches in finite time and blowing up of $u_{t}$ at the only quenching point $x=1$. First of all, we have the following:

Lemma 3.1 Assume that $\left(\mathrm{A}_{2}\right)$ and $\left(\mathrm{A}_{4}\right)$ hold and the solution $u$ of the problem (1.1) exists in $\left(0, \tilde{T}_{0}\right)$ for some $\tilde{T}_{0}>0$. Then $u_{x}(x, t)<0$ and $u_{t}(x, t)<0$ in $(0,1] \times\left(0, \tilde{T}_{0}\right)$.

The proof is similar to Lemma 2.1, so we omit it.

Theorem 3.1 Assume that $\left(\mathrm{A}_{2}\right)$ and $\left(\mathrm{A}_{4}\right)$ hold. Then there exists a finite time $T$, such that every solution of (1.1) quenches in this time, and the only quenching point is $x=1$.

Proof By the maximum principle, we can obtain $0<u(\cdot, t)<1$ for all $t$ in the existence interval. Together with $\left(\mathrm{A}_{4}\right)$, we get

$$
\beta=-u^{-q(p-1)}(1,0)+\int_{0}^{1}(1-u(x, 0))^{-h} d x<0 .
$$

Denote $I(t)=\int_{0}^{1} u(x, t) d x$. By Lemma 3.1, it is easy to see that

$$
I^{\prime}(t)=\int_{0}^{1} u_{t}(x, t) d x=-u^{-q(p-1)}(1, t)-+\int_{0}^{1}(1-u(x, t))^{-h} d x \leq \beta
$$

Thus $I(t) \leq I(0)+\beta t$, which means that $I\left(\tilde{t_{0}}\right)=0$ for some $\tilde{t_{0}}>0$. In addition, notice that $u_{x}<0$ for $0<x \leq 1$, we can see that there exists $T\left(0<T<\tilde{t_{0}}\right)$ such that $\lim _{t \rightarrow T^{-}} u(1, t)=0$. Combining with the singular nonlinearity of the boundary flux, $u$ must occur quenching on the boundary $x=1$. As in Theorem 2.1, in the following, we only need to show that the solutions $u$ cannot take place quenching in $(1 / 2,1) \times(\gamma, T)$ for some $\gamma(0<\gamma<T)$.

Define

$$
H(x, t)=u_{x}+\varepsilon\left(x-\frac{1}{4}\right), \quad(x, t) \in\left(\frac{1}{4}, 1\right) \times(\gamma, T),
$$

where $\varepsilon$ is sufficiently small. Since $u_{x}(x, t)<0$ in $(0,1] \times[0, T), H(x, t)$ satisfies

$$
\begin{aligned}
& H_{t}-(p-1)\left|u_{x}\right|^{p-2} H_{x x}=-(p-1)(p-2)\left|u_{x}\right|^{p-3}\left(H_{x}-\varepsilon\right)^{2}+h(1-u)^{-h-1} u_{x}<0, \\
& \quad \text { for }(x, t) \in\left(\frac{1}{4}, 1\right) \times(\gamma, T) .
\end{aligned}
$$

Further, on the parabolic boundary, since $u_{x}(x, t)<0$ in $(0,1] \times[0, T)$ and choosing $\varepsilon$ small enough, we have

$$
\begin{aligned}
& H\left(\frac{1}{4}, t\right)=u_{x}\left(\frac{1}{4}, t\right)<0, \quad \text { for } t \in[\gamma, T), \\
& H(1, t)=-u^{-q}(1, t)+\frac{3}{4} \varepsilon \leq-1+\frac{3}{4} \varepsilon<0, \quad \text { for } t \in[\gamma, T), \\
& H(x, \tilde{\eta}) \leq u_{x}\left(\frac{1}{4}, \gamma\right)+\frac{3}{4} \varepsilon<0, \quad \text { for } x \in\left[\frac{1}{4}, 1\right] .
\end{aligned}
$$


Making use of the maximum principle, we obtain $H(x, t) \leq 0$ in $(1 / 4,1) \times(\gamma, T)$, which yields

$$
-u_{x} \geq \varepsilon\left(x-\frac{1}{4}\right), \quad(x, t) \in\left(\frac{1}{4}, 1\right) \times(\tilde{\eta}, T) .
$$

Integrating (3.1) with respect to $x$ from $x$ to 1 gives

$$
u(x, t) \geq u(1, t)+\int_{x}^{1} \varepsilon\left(x-\frac{1}{4}\right) d x \geq \int_{x}^{1} \varepsilon\left(x-\frac{1}{4}\right) d x>0
$$

which implies that $u(x, t)$ if $x<1$. This completes the proof of Theorem 3.1.

Theorem $3.2 u_{t}$ blows up at the quenching point $x=1$.

Proof We prove the theorem by contradiction. Assume that $u_{t}$ is bounded on $[0,1] \times$ $[0, T)$. Then there exists a positive constant $L$ such that $u_{t}>-L$. Thus, we have

$$
\left(\left|u_{x}\right|^{p-2} u_{x}\right)_{x}+(1-u)^{-h}>-L
$$

Integrating with respect to $x$ from $x$ to 1 yields

$$
-u^{-q(p-1)}(1, t)>-u^{-q(p-1)}(x, t)-L-(1-u(0, t))^{-h}
$$

Therefore, it is found that the left-hand side tends to negative infinity as $t \rightarrow T^{-}$, while the right-hand side is finite. This completes the proof of Theorem 3.2.

\subsection{Quenching rate}

Now, we are in a position to investigate the bounds on the quenching rate. First of all, we will show the lower bound of the quenching rate.

Theorem 3.3 Assume that $\left(\mathrm{A}_{2}\right)$ and $\left(\mathrm{A}_{4}\right)$ hold. Then there exists a positive constant $C_{2}$ such that

$$
u(1, t) \geq C_{2}(T-t)^{\frac{1}{p q+2}}
$$

for $t$ sufficiently close to $T$.

Proof Let $k(u)=-q u^{-q(p-1)(\delta-1)-q-1}$, where $1-\frac{q+1}{q(p-1)}<\delta<1-\frac{1}{q(p-1)}$. It is easy to see that $k(u)<0, k^{\prime}(u)>0$, and $k^{\prime \prime}(u)<0$. Letting $\tau$ be close to $T$, we introduce the function

$$
Q(x, t)=u_{t}-\varepsilon k(u)\left(-u_{x}\right)^{(p-1)(2-\delta)}, \quad \text { in }(1-T+\tau, 1) \times(\tau, T),
$$

where $\varepsilon$ is a positive constant. Through a fairly complicated calculation, one has

$$
Q_{t}=(p-1)\left|u_{x}\right|^{p-2} Q_{x x}+(p-1)(p-2)\left(-u_{x}\right)^{p-3}\left(-u_{x x}\right) Q_{x}+J(x, t) Q+W(x, t) .
$$


Here

$$
\begin{aligned}
J(x, t)= & h(1-u)^{-h-1}+\varepsilon(2-\delta)[(p-1)(2-\delta)-1]\left(-u_{x}\right)^{(p-1)(1-\delta)-1} k(u) u_{t} \\
& +\varepsilon[(p-1)(5-2 \delta)-1] k^{\prime}(u)\left(-u_{x}\right)^{(p-1)(2-\delta)} \\
& +\varepsilon^{2}(2-\delta)[(p-1)(2-\delta)-1] k^{2}(u)\left(-u_{x}\right)^{(p-1)(3-2 \delta)-1}
\end{aligned}
$$

and

$$
\begin{aligned}
W(x, t)= & \varepsilon(p-1) k^{\prime \prime}(u)\left(-u_{x}\right)^{(p-1)(3-\delta)+1}+\varepsilon^{2}[(p-1)(5-2 \delta)-1] k^{\prime}(u) k(u)\left(-u_{x}\right)^{2(p-1)(2-\delta)} \\
& +\varepsilon^{3}(2-\delta)[(p-1)(2-\delta)-1] k^{3}(u)\left(-u_{x}\right)^{(p-1)(5-3 \delta)-1} \\
& -2 \varepsilon(2-\delta)[(p-1)(2-\delta)-1] k(u)\left(-u_{x}\right)^{(p-1)(1-\delta)-1} u_{t}(1-u)^{-h} \\
& +\varepsilon(2-\delta)[(p-1)(2-\delta)-1] k(u)\left(-u_{x}\right)^{(p-1)(1-\delta)}(1-u)^{-2 h} \\
& -\varepsilon(p-1)\left[(5-2 \delta) k^{\prime}(u)(1-u)+(2-\delta) k(u) h\right]\left(-u_{x}\right)^{(p-1)(2-\delta)}(1-u)^{-h-1}
\end{aligned}
$$

Notice that $k(u)<0, k^{\prime}(u)>0, k^{\prime \prime}(u)<0$, and $\tau$ is sufficiently close to $T$, then $J(x, t)>0$ and $W(x, t)<0$. Therefore,

$$
\begin{aligned}
& Q_{t}<(p-1)\left|u_{x}\right|^{p-2} Q_{x x}+(p-1)(p-2)\left(-u_{x}\right)^{p-3}\left(-u_{x x}\right) Q_{x}+J(x, t) Q, \\
& \quad(x, t) \in(1-T+\tau, 1) \times(\tau, T) .
\end{aligned}
$$

Further, on the parabolic boundary, in view of the only quenching point $x=1$ and provided $\varepsilon$ sufficient small, both $Q(1-T+\tau, t)$ and $Q(x, \tau)$ are negative. On the right boundary $x=1$, we get

$$
\begin{aligned}
Q_{x}(1, t)= & q[1-\varepsilon(2-\delta)] u^{-q-1}(1, t) Q(1, t)+\varepsilon q\{[\varepsilon q(2-\delta)+q(p-1)(\delta-1)+1] \\
& \left.\times u^{-q p-1}(1, t)+(2-\delta)(1-u(1, t))^{-h}\right\} u^{-q-1}(1, t) \\
\leq & q[1-\varepsilon(2-\delta)] u^{-q-1}(1, t) Q(1, t),
\end{aligned}
$$

provided $\varepsilon$ is sufficiently small and $\tau$ is sufficiently close to $T$. Thus, take advantage of the maximum principle, $Q(x, t) \leq 0$ on $[1-T+\tau, 1] \times[\tau, T)$. Then we have $Q(1, t) \leq 0$, that is,

$$
u_{t}(1, t) \leq \varepsilon k(u(1, t))\left(-u_{x}(1, t)\right)^{(p-1)(2-\delta)}=-\varepsilon q u^{-q p-1}(1, t) .
$$

Integrating (3.2) with respect to $t$ from $t$ to $T$, it gives

$$
u(1, t) \geq[\varepsilon q(q p+2)]^{\frac{1}{q p+2}}(T-t)^{\frac{1}{q p+2}}=C_{2}(T-t)^{\frac{1}{q p+2}},
$$

where $C_{2}=[\varepsilon q(q p+2)]^{\frac{1}{q p+2}}$. This completes the proof of Theorem 3.3.

To end this section, we present the upper bound on the quenching rate. 
Theorem 3.4 Assume that $\left(\mathrm{A}_{2}\right)$ and $\left(\mathrm{A}_{4}\right)$ hold. Then there exists a positive constant $C_{3}$ such that

$$
u(1, t) \leq C_{3}(T-t)^{\frac{1}{p q+2}}
$$

for $t$ sufficiently close to $T$.

Proof Denote $E(x, t)=\left|u_{x}(x, t)\right|^{p-2} u_{x}(x, t)+\varrho^{p-1}(x) u^{-q(p-1)}(x, t)$ in $(0,1) \times(0, T)$, where

$$
\varrho(x)= \begin{cases}0, & x \in\left[0, x_{0}\right], \\ \frac{\left(x-x_{0}\right)^{r}}{\left(1-x_{0}\right)^{r}}, & x \in\left(x_{0}, 1\right],\end{cases}
$$

with some $x_{0}<1$ and choosing $r \geq 3$ large enough so that $\varrho(x) \leq-u_{0}^{\prime}(x) u_{0}^{q}(x)$ for $x_{0}<x \leq 1$. We can easily obtain $E(0, t)=E(1, t)=0$, and $E(x, 0) \leq 0$. In addition, in $(0,1) \times(0, T)$, E satisfies

$$
\begin{aligned}
E_{t}= & (p-1)\left|u_{x}\right|^{p-2} E_{x x} \\
& -(p-1)^{2}\left[p q^{2}+q\right] \varrho^{p-1}(x)\left|u_{x}\right|^{p} u^{-q(p-1)-2} \\
& -2(p-1)^{3} q \varrho^{p-2}(x) \varrho^{\prime}(x)\left|u_{x}\right|^{p-1} u^{-q(p-1)-1} \\
& -(p-1)^{2}\left|u_{x}\right|^{p-2} \varrho^{p-3}(x)\left[(p-2) \varrho^{\prime 2}(x)+\varrho(x) \varrho^{\prime \prime}(x)\right] u^{-q(p-1)} \\
& -q(p-1) \varrho^{p-1}(x) u^{-q(p-1)-1}(1-u)^{-h}-(p-1) h\left|u_{x}\right|^{p-1}(1-u)^{-h-1} .
\end{aligned}
$$

According to the definition of $\varphi(x)$, it is easy to see that $\varrho(x) \geq 0, \varrho^{\prime}(x) \geq 0$, and $\varrho^{\prime \prime}(x) \geq 0$. Then we have

$$
E_{t} \leq(p-1)\left|u_{x}\right|^{p-2} E_{x x}
$$

Making use of the maximum principle, we get $E(x, t) \leq 0$, that is,

$$
\varrho(x) u^{-q}(x, t) \leq-u_{x}(x, t), \quad \text { for }(x, t) \in[0,1] \times[0, T) .
$$

Furthermore, because of $E(x, t) \leq 0$, we have $E_{x}(1, t) \geq 0$. In fact,

$$
E_{x}(1, t)=\lim _{x \rightarrow 1^{-}} \frac{E(x, t)-E(1, t)}{x-1} \geq 0,
$$

which implies

$$
\begin{aligned}
u_{t}(1, t) & \geq-(p-1)\left[\varrho^{\prime}(1)+q u^{-q-1}(1, t)\right] u^{-q(p-1)}(1, t)+(1-u(1, t))^{-h} \\
& \geq-\tilde{C} q(p-1) u^{-p q-1}(1, t) .
\end{aligned}
$$

Integrating (3.3) with respect to $t$ from $t$ to $T$ gives

$$
u(1, t) \leq[\tilde{C} q(p-1)(p q+2)]^{\frac{1}{p q+2}}(T-t)^{\frac{1}{p q+2}}=C_{3}(T-t)^{\frac{1}{p q+2}},
$$


where $C_{3}=[\tilde{C} q(p-1)(p q+2)]^{\frac{1}{p q+2}}$, which produces the asserted result. This completes the proof of Theorem 3.4 .

From Theorem 3.3 and Theorem 3.4, we have the following exact quenching rate.

Corollary 3.1 Assume that $\left(\mathrm{A}_{2}\right)$ and $\left(\mathrm{A}_{4}\right)$ hold. Then the solution of the problem (1.1) satisfies

$$
C_{2}(T-t)^{\frac{1}{p q+2}} \leq u(1, t) \leq C_{3}(T-t)^{\frac{1}{p q+2}}
$$

that is,

$$
u(1, t) \sim(T-t)^{\frac{1}{p q+2}}
$$

for t sufficiently close to T. Here $C_{2}, C_{3}$ are positive constants which are given in Theorem 3.3 and Theorem 3.4.

\section{Competing interests}

The author declares to have no competing interests.

\section{Acknowledgements}

This work is partially supported by the National Science Foundation of China (11201311, 11301345), Guangdong Natural Science Foundation (2014A030310074) and Natural Science Foundation of SZU $(201425,201545)$. The author would like to thank the referees for revising the manuscript and for many valuable comments, which helped to clarify this paper.

Received: 1 August 2015 Accepted: 29 November 2015 Published online: 10 December 2015

\section{References}

1. Kawarada, H: On solutions of initial-boundary problem $u_{t}=u_{x x}+1 /(1-u)$. Publ. Res. Inst. Math. Sci. 10, $729-736$ (1975)

2. Fila, M, Levine, HA: Quenching on the boundary. Nonlinear Anal. TMA 21, 795-802 (1993)

3. Levine, HA: The quenching of solutions of linear parabolic and hyperbolic equations with nonlinear boundary conditions. SIAM J. Math. Anal. 14, 1139-1153 (1983)

4. Levine, HA: Quenching, nonquenching, and beyond quenching for solutions of some parabolic equations. Ann. Mat. Pura Appl. 155, 243-260 (1989)

5. Ferreira, R, Pablo, AD, Quirós, F, Rossi, JD: Non-simultaneous quenching in a system of heat equations coupled on the boundary. Z. Angew. Math. Phys. 57, 1-9 (2006)

6. Jin, CH, Yin, JX, Zhang, XP: Critical quenching exponents for heat equations coupled with nonlinear boundary flux. Northeast. Math. J. 25, 88-96 (2009)

7. Chan, CY, Yuen, SI: Parabolic problems with nonlinear absorptions and releases at the boundaries. Appl. Math. Comput. 121(2-3), 203-209 (2001)

8. Selcuk, B, Ozalp, N: The quenching behavior of a semilinear heat equation with a singular boundary outflux. Q. Appl. Math. 72(4), 747-752 (2014)

9. Zhi, YH, Mu, CL: The quenching behavior of a nonlinear parabolic equation with nonlinear boundary outflux. Appl. Math. Comput. 184(2), 624-630 (2007)

10. Zhao, CL: Blow-up and quenching for solutions of some parabolic equations. Ph.D. thesis, University of Louisiana, Lafayette (2000)

11. Ozalp, N, Selcuk, B: Blow up and quenching for a problem with nonlinear boundary conditions. Electron. J. Differ. Equ. 2015, 192 (2015)

12. Ozalp, N, Selcuk, B: The quenching behavior of a nonlinear parabolic equation with a singular boundary condition. Hacet. J. Math. Stat. 44(3), 615-621 (2015)

13. Deng, K: Quenching for solutions of a plasma type equation. Nonlinear Anal. TMA 18, 731-742 (1992)

14. Nie, YY, Wang, CP, Zhou, Q: Quenching for singular and degenerate quasilinear diffusion equations. Electron. J. Differ. Equ. 2013, 13 (2013)

15. Fila, M, Kawohl, B, Levine, HA: Quenching for quasilinear equations. Commun. Partial Differ. Equ. 17, 593-614 (1992)

16. Deng, $\mathrm{K}, \mathrm{Xu}, \mathrm{M}$ : Quenching for a nonlinear diffusion equation with a singular boundary condition. Z. Angew. Math. Phys. 50, 574-584 (1999)

17. Zhou, MJ, Wang, CP, Nie, YY: Quenching of solutions to a class of semilinear parabolic equations with boundary degeneracy. J. Math. Anal. Appl. 421(1), 59-74 (2015)

18. Yang, Y, Yin, JX, Jin, CH: A quenching phenomenon for one-dimensional $p$-Laplacian with singular boundary flux. Appl. Math. Lett. 23(9), 955-959 (2010)

19. Yang, Y, Yin, JX, Jin, CH: Quenching phenomenon of positive radial solutions for $p$-Laplacian with singular boundary flux. J. Dyn. Control Syst. (2015). doi:10.1007/s10883-015-9287-2 